Pacific

Journal of

Mathematics

\title{
CURVES WITH PRESCRIBED INTERSECTION WITH BOUNDARY DIVISORS IN MODULI SPACES OF CURVES
}

XiAO-LeI LiU 


\title{
CURVES WITH PRESCRIBED INTERSECTION WITH BOUNDARY DIVISORS IN MODULI SPACES OF CURVES
}

\author{
XiAO-LEI LiU
}

\begin{abstract}
We construct curves in moduli spaces of curves with prescribed intersection with boundary divisors. As applications, we obtain families of curves with maximal slope as well as extremal test curves for the weakly positive cone of the moduli space.
\end{abstract}

\section{Introduction}

Let $M_{g}$ be the moduli space of smooth curves of genus $g$ over the field of complex numbers $\mathbb{C}$, and $\Delta_{0}, \Delta_{1}, \ldots, \Delta_{[g / 2]}$ the boundary divisors of the Deligne-Mumford compactification $\bar{M}_{g}$. Denote by $\mathscr{H}_{g}$ the moduli space of smooth hyperelliptic curves of genus $g$; then the restriction of $\Delta_{0}$ to the closure $\overline{\mathscr{H}}_{g}$ breaks up into $\Xi_{0}, \Xi_{1}, \ldots, \Xi_{[(g-1) / 2]}$. The restriction of $\Delta_{i}$ to $\overline{\mathscr{H}}_{g}$ is often denoted by $\Theta_{i}$ for $i>0$; see [Harris and Morrison 1998].

A family of curves of genus $g$ over a curve $Y$ is a fibration $f: X \rightarrow Y$ whose general fibers are smooth curves of genus $g$, where $X$ is a smooth projective surface. Let $\omega_{X / Y}$ be the relative dualizing sheaf. If $f$ is nontrivial, the slope of $f$ is $\omega_{X / Y}^{2} / \operatorname{deg} f_{*} \omega_{X / Y}$. Let $J_{f}: Y \rightarrow \bar{M}_{g}$ denote the moduli map induced by $f$; see [Tan 2010].

Special curves in moduli spaces play an important rule in the study of birational geometry of moduli spaces: for example, the ample cone, the nef cone and the Mori cone of curves [Gibney 2009]. Before raising our problems, we firstly summarize some interesting properties of curves in moduli spaces with prescribed intersection with boundary divisors. In this paper, we always assume that curves in moduli spaces are complete irreducible and are not contained in boundary divisors.

If $C=J_{f}(Y) \subset \overline{\mathscr{H}}_{g}$ is a curve intersecting only $\Xi_{0}$ (resp. $\Delta_{[g / 2]}$ ), then the semistable reduction of $f$ has minimal (resp. maximal) slope; see [Liu 2016, Remark 3.9] and [Liu and Tan 2013, Theorem 3.1]. We refer to [Tan 2010] for related discussions. Moreover, if $C=J_{f}(Y) \subset \bar{M}_{g}$ is disjoint from boundary divisors, then the semistable reduction of $f$ is a Kodaira fibration; see [Kodaira 1967].

MSC2010: 14D22.

Keywords: intersection, boundary divisors, moduli spaces of curves. 
On the other hand, curves intersecting exactly one boundary divisor of $\overline{\mathscr{H}}_{g}$ are the extremal test curves of the weakly positive cone in $\operatorname{Pic}\left(\bar{M}_{g}\right) \otimes \mathbb{Q}$. Here the weakly positive cone consists of weakly positive $\mathbb{Q}$-Cartier divisors over $\mathcal{M}_{g}$; see Section 1 in [Moriwaki 1998] for the definition of weakly positive divisors, and Section 4 of that paper for this result.

Motivated by these properties, we want to study further properties of curves that intersect given boundary divisors. In this paper we discuss the existence of such curves.

Let $C$ be a curve in $\overline{\mathscr{H}}_{g}$, and $\mathscr{B}=\left\{\Theta_{1}, \ldots, \Theta_{[g / 2]}, \Xi_{0}, \Xi_{1}, \ldots, \Xi_{[(g-1) / 2]}\right\}$ be

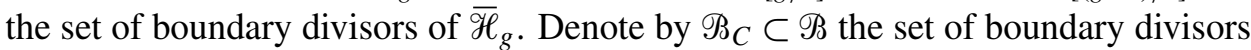
of $\overline{\mathscr{H}}_{g}$ that intersect $C$.

Problem 1.1. For any nonempty subset $\mathscr{B}^{\prime} \subseteq \mathscr{B}$, does there exist a curve $C$ in $\overline{\mathscr{H}}_{g}$ such that the boundary divisors of $\overline{\mathscr{H}}_{g}$ that intersect $C$ are those in $\mathscr{B}^{\prime}$, i.e., $\mathscr{B}_{C}=\mathscr{B}^{\prime}$ ?

Let $\widetilde{\mathcal{M}}_{0, n}$ be the moduli space of stable unordered $n$-pointed rational curves. Let $B_{k}$ be the boundary divisor of $\widetilde{M}_{0, n}$ whose general point parametrizes the union of a $k$-pointed $\mathbb{P}^{1}$ and an $(n-k)$-pointed $\mathbb{P}^{1}$ for $2 \leq k \leq[n / 2]$.

One can regard $\overline{\mathscr{H}}_{g}$ as the Hurwitz space parametrizing genus $g$ admissible double covers of rational curves. Such a cover uniquely corresponds to a stable $(2 g+2)$-pointed rational curve by marking the branch points of the cover. Thus $\overline{\mathscr{H}}_{g}$ can be further identified as $\widetilde{\mathcal{M}}_{0,2 g+2}$. The natural isomorphism $\overline{\mathscr{H}}_{g} \cong \widetilde{M}_{0,2 g+2}$ induces the identifications $\Xi_{i}=B_{2 i+2}$ and $\Theta_{i}=B_{2 i+1}$. Also denote by $\mathscr{B}=$ $\left\{B_{2}, B_{3}, \ldots, B_{[n / 2]}\right\}$ the set of boundary divisors of $\widetilde{\mathcal{M}}_{0, n}$. Hence the existence of curves in $\overline{\mathscr{H}}_{g}$ in Problem 1.1 is the same as that in $\widetilde{\mathcal{M}}_{0, n}$ for $n=2 g+2$. For the sake of completeness, we consider the existence of curves in $\widetilde{M}_{0, n}$. Precisely, we consider the following problem.

Problem 1.2. For any nonempty subset $\mathscr{B}^{\prime} \subseteq \mathscr{B}$, does there exist a curve $C$ in $\widetilde{M}_{0, n}$ such that the boundary divisors of $\widetilde{M}_{0, n}$ that intersect $C$ are those in $\mathscr{B}^{\prime}$, i.e., such that $\mathscr{B}_{C}=\mathscr{B}^{\prime}$ ?

The purpose of this paper is to answer these problems in a number of cases. We have the following uniform solution for small $n$.

Theorem 1.3. Assume $n \leq 17$. For any nonempty subset $\mathscr{B}^{\prime}$ of $\mathscr{B}$, there exists a curve $C$ in $\widetilde{M}_{0, n}$ such that $\mathscr{B}_{C}=\mathscr{B}^{\prime}$.

Unfortunately, our method is invalid for $n=18$ (see Remark 6.1).

Corollary 1.4. For $2 \leq g \leq 7$ and any nonempty $\mathscr{B}^{\prime} \subset \mathscr{B}$, there exists a curve $C$ in $\overline{\mathscr{H}}_{g}$ such that $\mathscr{B}_{C}=\mathscr{B}^{\prime}$.

Corollary 1.5. For $2 \leq g \leq 7$ and any nonempty $\mathscr{B}^{\prime} \subset \Delta=\left\{\Delta_{0}, \Delta_{1}, \ldots, \Delta_{[g / 2]}\right\}$, there exists a curve $C$ in $\bar{M}_{g}$ with $\mathscr{B}_{C}=\mathscr{B}^{\prime}$. 
In order to simplify the proof of the above theorem, we use the following two partial solutions of Problem 1.2.

Theorem 1.6. Let $\mathscr{B}^{\prime}$ be a subset of the set $\mathscr{B}=\left\{B_{2}, \ldots, B_{[n / 2]}\right\}$ of boundary divisors of $\widetilde{M}_{0, n}$.

(1) For $\left|\mathscr{B}^{\prime}\right|=1$, if $\mathscr{B}^{\prime}=\left\{B_{i}\right\}$, then there exists a curve $C$ in $\widetilde{M}_{0, n}$ such that $\mathscr{B}_{C}=\mathscr{B}^{\prime}$.

(2) For $\left|\mathscr{B}^{\prime}\right|=2$, if $\mathscr{B}^{\prime}=\left\{B_{i}, B_{i+1}\right\}$ or $\mathscr{B}^{\prime}=\left\{B_{i}, B_{i+2}\right\}$, then there exists a curve $C$ in $\widetilde{M}_{0, n}$ such that $\mathscr{B}_{C}=\mathscr{B}^{\prime}$.

(3) For $\left|\mathscr{B}^{\prime}\right|=3$, if $\mathscr{B}^{\prime}$ is one of $\left\{B_{i}, B_{j}, B_{i+j+1}\right\},\left\{B_{i}, B_{j}, B_{i+j}\right\},\left\{B_{i}, B_{j}, B_{i+j-1}\right\}$, or $\left\{B_{i}, B_{j}, B_{i+j-2}\right\}$, then there exists a curve $C$ in $\widetilde{M}_{0, n}$ such that $\mathscr{B}_{C}=\mathscr{M}^{\prime}$.

In particular, we recover the existence of a hyperelliptic family with maximal slope from [Liu and Tan 2013], where the authors construct explicit polynomials to define the family. As another application, we also give an alternative proof of the existence of extremal test curves for the weakly positive cone from Appendix A in [Moriwaki 1998], where the author uses the existence of a concrete linear system. Here we reduce the problem to the existence of rational functions and then give a unitive method. This new method greatly generalizes the former results.

Theorem 1.7. Let $\mathscr{B}^{\prime}$ be a subset of the set $\mathscr{B}=\left\{B_{2}, \ldots, B_{[n / 2]}\right\}$ of boundary divisors of $\widetilde{M}_{0, n}$.

(1) If $\mathscr{P}^{\prime}=\left\{B_{2}, B_{i_{1}}, \ldots, B_{i_{k}}\right\}$ and $\left(i_{1}-1\right)+\cdots+\left(i_{k}-1\right) \leq n-2$, then there exists a curve $C$ in $\widetilde{M}_{0, n}$ such that $\mathscr{B}_{C}=\mathscr{B}^{\prime}$.

(2) If $\mathscr{B}^{\prime}=\left\{B_{2}, B_{k+1}, B_{k+2}, \ldots, B_{[n / 2]}\right\}$ and $2 \leq k \leq[n / 2]$, then there exists $a$ curve $C$ in $\widetilde{M}_{0, n}$ such that $\mathscr{B}_{C}=\mathscr{P}^{\prime}$.

(3) If $\mathscr{B}^{\prime}=\left\{B_{3}, B_{i_{1}}, \ldots, B_{i_{k}}\right\}$ and $\left(i_{1}-1\right)+\cdots+\left(i_{k}-1\right) \leq n-3$, then there exists a curve $C$ in $\widetilde{M}_{0, n}$ such that $\mathscr{B}_{C}=\mathscr{B}^{\prime}$.

Now we explain the main idea of the proofs. In order to construct curves in moduli spaces intersecting the given boundary divisors, we use the following three different methods:

(i) We regard a rational function $\phi$ of degree $n$ as a 1-dimensional family of unordered $n$ marked points in $\mathbb{P}^{1}$ in Section 3. Only the critical points of $\phi$ may correspond to points in boundary divisors; see the correspondence (3-1). Then we just need to construct rational functions with the desired critical points. The existence of such rational functions is from the main Theorem of [Scherbak 2002].

(ii) The graph $G_{\phi}$ of $\phi$ is a smooth rational curve in $\mathbb{P}^{1} \times E$, where $E \cong \mathbb{P}^{1}$. Let $p$ be the second projection and $R$ the reducible curve consisting of $G_{\phi}$ and certain sections of $p$. Then the restriction $\left.p\right|_{R}$ is a branched cover over $E$, and 
this cover induces different 1 -dimensional families of marked points in $\mathbb{P}^{1}$. The correspondence (4-1) gives a relation between points in boundary divisors with ramification points of $\left.p\right|_{R}$, i.e., between singular points of $R$ and ramification points of $\left.p\right|_{G_{\phi}}$. So we only need to construct curves $R$ with suitable ramification points in Section 4 . This method generalizes method (i), and both methods are effective for many cases.

On the other hand, if $n$ is large, $\mathscr{B}^{\prime}$ may have so many elements that there is no desired rational function, rendering the above two methods invalid; see Remark 6.1. For this difficulty, we have the following method for special $\mathscr{B}^{\prime}$.

(iii) Taking the intersection of general very ample divisors of Hassett's weighted moduli spaces, we can obtain curves intersecting all the boundary divisors of these spaces. The proper transforms of these curves in $\widetilde{M}_{0, n}$ by the reduction morphism are also the curves we need; see Section 5.

\section{Existence of rational functions}

Let $\phi(x)=f(x) / g(x)$ be a rational function of degree $n$; i.e., $f(x)$ and $g(x)$ are polynomials without common roots and $n=\max \{\operatorname{deg} f(x), \operatorname{deg} g(x)\}$. A point $z \in \mathbb{C}$ is a critical point of multiplicity $m$ if $z$ is a root of the Wronskian $W(x)$ of $f(x)$ and $g(x)$ of multiplicity $m$, where

$$
W(x)=g^{2}(x) \phi^{\prime}(x)=f^{\prime}(x) g(x)-f(x) g^{\prime}(x) .
$$

Let all the finite critical points of $\phi(x)$ be denoted $z_{1}, \ldots, z_{l-1}$ with multiplicities $m_{1}, \ldots, m_{l-1}$, respectively. According to the Riemann-Hurwitz formula, $2 n-2 \geq$ $m_{1}+\cdots+m_{l-1}$. Let $z_{l}$ be the point at infinity; then the difference

$$
m_{l}=2 n-2-\left(m_{1}+\cdots+m_{l-1}\right)
$$

is the multiplicity of $\phi(x)$ at infinity. If $z_{1}, \ldots, z_{l-1}$ are in general position and $1 \leq m_{i} \leq n-1$ for each $1 \leq i \leq l$, then we say that $\phi(x)$ is a rational function of type $\left(n ; m_{1}, \ldots, m_{l}\right)$.

Note that, up to the point at infinity, the types of rational functions here are the same as in [Scherbak 2002].

If $\phi_{1}(x)$ and $\phi_{2}(x)$ are two rational functions satisfying

$$
\phi_{2}(x)=\frac{a \phi_{1}(x)+b}{c \phi_{1}(x)+d}, \quad a d-b c \neq 0,
$$

then $\phi_{1}(x)$ and $\phi_{2}(x)$ have the same type, and we say that $\phi_{1}(x)$ and $\phi_{2}(x)$ are in the same class of rational functions. 
Theorem 2.1 [Scherbak 2002]. Let $l \geq 3$ and $n \geq 2$ be integers, and also let $1 \leq m_{i} \leq n-1$ for $1 \leq i \leq l$ be integers satisfying (2-1). Then the number $\#\left(n ; m_{1}, \ldots, m_{l}\right)$ of classes of rational functions of type $\left(n ; m_{1}, \ldots, m_{l}\right)$ is

(2-2) \#(n; $\left.m_{1}, \ldots, m_{l}\right)=\sum_{q=1}^{l-1}(-1)^{l-1-q} \sum_{1 \leq i_{1}<\cdots<i_{q} \leq l-1}\left(\begin{array}{c}m_{i_{1}}+\cdots+m_{i_{q}}+q-n-1 \\ l-3\end{array}\right)$,

and any nonempty class can be represented by the ratio of polynomials without multiple roots.

Proof. Let $\boldsymbol{m}=\left(m_{1}, \ldots, m_{l-1}\right)$; then $\#\left(n ; m_{1}, \ldots, m_{l}\right)$ is equal to \# $(n, l-1 ; \boldsymbol{m})$ in the main theorem in [Scherbak 2002], from which the result directly follows.

As usual, we set $\left(\begin{array}{l}a \\ b\end{array}\right)=0$ for $a<b$.

\section{Corollary 2.2.}

(1) There exists a rational function $\phi(x)$ of type $\left(n ; m_{1}, \ldots, m_{l-1}, m_{l}=n-1\right)$.

(2) There exists a rational function $\phi(x)$ of type $\left(n ; m_{1}, m_{2}, m_{3}\right)$.

Proof. (1) Since $m_{l}=n-1$, we have $m_{1}+\cdots+m_{l-1}=n-1$, and the right side of $(2-2)$ is

$$
\left(\begin{array}{c}
m_{1}+\cdots+m_{l-1}+l-1-n-1 \\
l-3
\end{array}\right)=\left(\begin{array}{l}
l-3 \\
l-3
\end{array}\right)=1 .
$$

So the desired rational function exists by Theorem 2.1.

(2) If $m_{i}=n-1$ for some $i$, then the existence is from (1). We may assume that $m_{i} \leq n-2$ for $i=1,2,3$. Then the right side of (2-2) is

$$
\left(\begin{array}{c}
m_{1}+m_{2}+2-n-1 \\
0
\end{array}\right)-\left(\begin{array}{c}
m_{1}-n \\
0
\end{array}\right)-\left(\begin{array}{c}
m_{2}-n \\
0
\end{array}\right)=\left(\begin{array}{c}
m_{1}+m_{2}-n+1 \\
0
\end{array}\right)=1 .
$$

\section{Curves from rational functions}

Let $\phi(x)$ be a rational function of degree $n$; then it induces a degree $n$ branched cover $D \cong \mathbb{P}^{1} \rightarrow E \cong \mathbb{P}^{1}$. Varying a point $t \in E$, the union of the $n$ preimage points also varies in $D$; hence it provides a 1-dimensional family $E$ of unordered $n$ points in $\mathbb{P}^{1}$. So we obtain a curve in $\widetilde{M}_{0, n}$. When $t$ hits a branch point, suppose that over $t$ there is a ramification point $z_{i}$ locally of type $y=\phi(x)=x^{m_{i}+1}$, i.e., a critical point of $\phi(x)$ with multiplicity $m_{i}$. Making a degree $m_{i}+1$ base change, we then get an ordinary singularity of degree $m_{i}+1$. Blowing up the singularity separates the $m_{i}+1$ sheets, and thus $t$ corresponds to a point in the boundary component $B_{m_{i}+1}$ (or $B_{n-1-m_{i}}$ if $m_{i} \geq[n / 2]$, but not $m_{i}=n-1$ or $n-2$, which correspond to a point in the interior of $\left.\widetilde{\mathcal{M}}_{0, n}\right)$. Hence, if $\phi(x)$ is of type $\left(n ; m_{1}, \ldots, m_{l}\right)$ and $C$ is its corresponding curve in $\widetilde{\mathcal{M}}_{0, n}$, then

$$
\mathscr{B}_{C}=\left\{B_{m_{i}+1}: 1 \leq m_{i}<[n / 2]\right\} \cup\left\{B_{n-1-m_{i}}:[n / 2] \leq m_{i} \leq n-3\right\} .
$$


Theorem 3.1. Let $\mathscr{B}^{\prime}=\left\{B_{i_{1}}, \ldots, B_{i_{k}}\right\}$ be a subset of $\mathscr{B}=\left\{B_{2}, B_{3}, \ldots, B_{[n / 2]}\right\}$ with $\left(i_{1}-1\right)+\cdots+\left(i_{k}-1\right)=n-1$; then there exists a curve $C$ in $\widetilde{M}_{0, n}$ such that $\mathscr{B}_{C}=\mathscr{B}^{\prime}$. Moreover,

(1) if $\mathscr{B}^{\prime}=\left\{B_{2}, B_{i_{1}}, \ldots, B_{i_{k}}\right\}$, and $\left(i_{1}-1\right)+\cdots+\left(i_{k}-1\right) \leq n-2$, then there exists a curve $C$ in $\widetilde{M}_{0, n}$ such that $\mathscr{B}_{C}=\mathscr{B}^{\prime}$;

(2) if $\mathscr{B}^{\prime}=\left\{B_{3}, B_{i_{1}}, \ldots, B_{i_{k}}\right\}$, and $n-1-\left(i_{1}-1\right)-\cdots-\left(i_{k}-1\right)=2 j \geq 2$, then there exists a curve $C$ in $\widetilde{M}_{0, n}$ such that $\mathscr{S}_{C}=\mathscr{B}^{\prime}$.

Proof. If $\left(i_{1}-1\right)+\cdots+\left(i_{k}-1\right)=n-1$, set $i_{k+1}=n$; then there exists a rational function $\phi(x)$ of type $\left(n ; i_{1}-1, \ldots, i_{k}-1, i_{k+1}-1\right)$ by Corollary 2.2(1). Thus the curve $C \subset \widetilde{M}_{0, n}$ corresponding to $\phi(x)$ satisfies $\mathscr{B}_{C}=\left\{B_{i_{1}}, \ldots, B_{i_{k}}\right\}$ by (3-1).

(1) Let $h=n-1-\left(\left(i_{1}-1\right)+\cdots+\left(i_{k}-1\right)\right) \geq 1$ and $i_{k+1}=\cdots=i_{k+h}=2$; then $\left(i_{1}-1\right)+\cdots+\left(i_{k+h}-1\right)=n-1$, and hence there exists a curve $C \subset \widetilde{M}_{0, n}$ with $\mathscr{B}_{C}=\left\{B_{2}, B_{i_{1}}, \ldots, B_{i_{k}}\right\}$.

(2) If we take $i_{k+1}=\cdots=i_{k+j}=3$, then $\left(i_{1}-1\right)+\cdots+\left(i_{k+j}-1\right)=n-1$, and there exists a curve $C \subset \widetilde{M}_{0, n}$ with $\mathscr{B}_{C}=\left\{B_{3}, B_{i_{1}}, \ldots, B_{i_{k}}\right\}$.

Theorem 3.2. Let $\mathscr{B}^{\prime}$ be a subset of $\mathscr{B}=\left\{B_{2}, B_{3}, \ldots, B_{[n / 2]}\right\}$. If $\mathscr{B}^{\prime}$ is one of $\left\{B_{i}, B_{i+1}\right\},\left\{B_{i}, B_{i+2}\right\}$, or $\left\{B_{i}, B_{j}, B_{i+j+1}\right\}$, then there exists a curve $C$ in $\widetilde{M}_{0, n}$ with $\mathscr{B}_{C}=\mathscr{B}^{\prime}$.

Proof. If $\mathscr{R}^{\prime}=\left\{B_{i}, B_{i+1}\right\}$, then there exists a rational function $\phi_{1}(x)$ of type ( $n ; i, n-1-i, n-1)$ by Corollary 2.2(2). Then the curve $C \subset \widetilde{M}_{0, n}$ corresponding to $\phi_{1}(x)$ satisfies $\mathscr{B}_{C}=\left\{B_{i}, B_{i+1}\right\}$ by $(3-1)$.

By the same reasoning, for $\mathscr{B}^{\prime}=\left\{B_{i}, B_{i+2}\right\}$, there exists a rational function $\phi_{2}(x)$ of type $(n ; i+1, n-1-i, n-2)$, and the curve $C \subset \widetilde{M}_{0, n}$ corresponding to $\phi_{2}(x)$ satisfies $\mathscr{B}_{C}=\left\{B_{i}, B_{i+2}\right\}$.

Similarly, for $\mathscr{B}^{\prime}=\left\{B_{i}, B_{j}, B_{i+j+1}\right\}$, there exists a rational function $\phi_{3}(x)$ of type $(n ; n-1-i, n-1-j, i+j)$, and the curve $C \subset \widetilde{M}_{0, n}$ corresponding to $\phi_{3}(x)$ satisfies $\mathscr{B}_{C}=\left\{B_{i}, B_{j}, B_{i+j+1}\right\}$.

\section{Curves from rational functions and sections}

Let $\phi(x)$ be a rational function of type $\left(n-s ; m_{1}, \ldots, m_{l}\right)$, where $s<n$ is a nonnegative integer. Let $G_{\phi}$ be its graph in $\mathbb{P}^{1} \times E$, where $E \cong \mathbb{P}^{1}$; then $G_{\phi}$ is a smooth rational curve. We now consider the reducible curve

$$
R_{\phi, \Gamma_{1}, \ldots, \Gamma_{s}}=G_{\phi}+\Gamma_{1}+\cdots+\Gamma_{s},
$$

where the $\Gamma_{i}$ are sections of the second projection

$$
p: \mathbb{P}^{1} \times E \rightarrow E, \quad p((x, t))=t .
$$


So the restriction of $p$ to $R_{\phi, \Gamma_{1}, \ldots, \Gamma_{s}}$

$$
p: R_{\phi, \Gamma_{1}, \ldots, \Gamma_{s}} \rightarrow E
$$

is a cover of degree $n$. Similarly as in Section 3, varying a point $t \in E$, we obtain a 1-dimensional family of unordered $n$ marked points in $\mathbb{P}^{1}$. Hence we get a curve $C$ in $\widetilde{M}_{0, n}$. Note that this construction is the same as that in Section 3 when $s=0$.

Let $S_{1}=\left(\Gamma_{1}+\cdots+\Gamma_{s}\right) \cap G_{\phi}$, and let $S_{2}=\left\{z_{1}, \ldots, z_{l}\right\}$ be the set of all the critical points of $\phi(x)$, including the point at infinity. Here we identify the critical point $z_{i}$ of $\phi$ with its image $\left(z_{i}, \phi\left(z_{i}\right)\right)$ in $G_{\phi}$.

If $z \in S_{2} \cap S_{1}$, then the local equation of $p$ at $z$ is $x\left(x^{m+1}+t\right)=0$, where $m$ is the multiplicity of $z$. Making a degree $m+1$ base change

$$
t \mapsto u^{m+1},
$$

we get an ordinary singularity of degree $m+2$. Blowing it up, we then see that $p(z) \in E$ corresponds to a point in the boundary component $B_{m+2}$ (or $B_{n-2-m}$ if [n/2] $-1 \leq m \leq n-4$, but not if $m=n-3$ or $n-2$, corresponding to a point in the interior of $\widetilde{M}_{0, n}$ ).

If $z \in S_{2}-S_{1}$, then the point in $\widetilde{M}_{0, n}$ corresponding to $p(z)$ has been considered in Section 3. Hence we have that

$$
\begin{aligned}
\mathscr{B}_{C}=\left\{B_{2}: z \in S_{1} \backslash S_{2}\right\} & \cup\left\{B_{m_{i}+1}: 1 \leq m_{i}<\left[\frac{1}{2} n\right], z_{i} \in S_{2} \backslash S_{1}\right\} \\
& \cup\left\{B_{n-1-m_{i}}:\left[\frac{1}{2} n\right] \leq m_{i} \leq n-3, z_{i} \in S_{2} \backslash S_{1}\right\} \\
& \cup\left\{B_{m_{i}+2}: 1 \leq m_{i}<\left[\frac{1}{2} n\right]-1, z_{i} \in S_{2} \cap S_{1}\right\} \\
& \cup\left\{B_{n-2-m_{i}}:\left[\frac{1}{2} n\right]-1 \leq m_{i} \leq n-4, z_{i} \in S_{2} \cap S_{1}\right\} .
\end{aligned}
$$

Since we have considered the case that $\mathscr{B}^{\prime}$ contains $B_{2}$ in Theorem 3.1(1), we now consider the case where each section passes through a critical point of $\phi(x)$, that is, where $S_{1} \subset S_{2}$.

Theorem 4.1. Let $\mathscr{B}^{\prime}=\left\{B_{3}, B_{i_{1}}, \ldots, B_{i_{k}}\right\}$ be a subset of $\mathscr{B}=\left\{B_{2}, B_{3}, \ldots, B_{[n / 2]}\right\}$ with $n-1-\left(\left(i_{1}-1\right)+\cdots+\left(i_{k}-1\right)\right)=2 j+1 \geq 3$; then there exists a curve $C$ in $\widetilde{M}_{0, n}$ such that $\mathscr{B}_{C}=\mathscr{B}^{\prime}$.

Proof. Set $i_{k+1}=\cdots=i_{k+j}=3$, then

$$
\left(i_{1}-1\right)+\cdots+\left(i_{k}-1\right)+(3-1) j+((n-1)-1)=2((n-1)-1) .
$$

Hence there exists a rational function $\phi(x)$ of type $\left(n-1 ; i_{1}-1, \ldots, i_{k+j}-1, n-2\right)$ by Corollary 2.2(1). Let $\Gamma$ be the section passing through the critical point $z_{k+j+1}$, where the multiplicity of $z_{k+j+1}$ of $\phi(x)$ is $n-2$. Thus the reducible curve $R_{\phi, \Gamma}=$ $G_{\phi}+\Gamma$ induces a curve $C \subset \widetilde{M}_{0, n}$ with $\mathscr{B}_{C}=\mathscr{B}^{\prime}=\left\{B_{3}, B_{i_{1}}, \ldots, B_{i_{k}}\right\}$ by (4-1). 
Corollary 4.2. If $\mathscr{P}^{\prime}=\left\{B_{3}, B_{i_{1}}, \ldots, B_{i_{k}}\right\}$, and $n-3-\left(\left(i_{1}-1\right)+\cdots+\left(i_{k}-1\right)\right) \geq 0$, then there exists a curve $C$ such that $\mathscr{B}_{C}=\mathscr{B}^{\prime}$.

Proof. If $n-3-\left(\left(i_{1}-1\right)+\cdots+\left(i_{k}-1\right)\right)$ is even, then it follows directly from Theorem 3.1(2); if $n-3-\left(\left(i_{1}-1\right)+\cdots+\left(i_{k}-1\right)\right)$ is odd, then it is from Theorem 4.1.

Theorem 4.3. Let $\mathscr{B}^{\prime}$ be a subset of $\mathscr{B}=\left\{B_{2}, B_{3}, \ldots, B_{[n / 2]}\right\}$. If $\mathscr{B}^{\prime}$ is one of $\left\{B_{i}\right\}$, $\left\{B_{i}, B_{j}, B_{i+j-2}\right\},\left\{B_{i}, B_{j}, B_{i+j}\right\}$, or $\left\{B_{i}, B_{j}, B_{i+j-1}\right\}$, then there exists a curve $C$ in $\widetilde{M}_{0, n}$ such that $\mathscr{B}_{C}=\mathscr{B}^{\prime}$.

Proof. Let $\phi_{1}(x)$ be a rational function of type $(n-1 ; i-1, n-i-1, n-2)$ and $\Gamma_{1}$ the section passing through the critical point $z_{3}$ of $\phi_{1}(x)$. Then the curve $C \subset \widetilde{M}_{0, n}$ induced by $R_{\phi_{1}, \Gamma_{1}}$ satisfies $\mathscr{B}_{C}=\left\{B_{i}\right\}$ by (4-1).

Let $\phi_{2}(x)$ be a rational function of type $(n-3 ; n-i-2, n-j-2, i+j-4)$ and $\Gamma_{2 i}$ the section passing through the critical point $z_{i}(i=1,2,3)$ of $\phi_{2}(x)$. Then the curve $C \subset \widetilde{M}_{0, n}$ induced by $R_{\phi_{2}, \Gamma_{21}, \Gamma_{22}, \Gamma_{23}}$ satisfies $\mathscr{B}_{C}=\left\{B_{i}, B_{j}, B_{i+j-2}\right\}$ by (4-1).

Let $\phi_{3}(x)$ be a rational function of type $(n-2 ; n-2-i, n-2-j, i+j-2)$ and $\Gamma_{3 i}$ the section passing through the critical point $z_{i}(i=1,2)$ of $\phi_{3}(x)$. Then the curve $C \subset \widetilde{M}_{0, n}$ induced by $R_{\phi_{3}, \Gamma_{31}, \Gamma_{32}}$ satisfies $\mathscr{B}_{C}=\left\{B_{i}, B_{j}, B_{i+j-1}\right\}$ by (4-1).

Let $\phi_{4}(x)$ be a rational function of type $(n-1 ; n-1-i, n-1-j, i+j-2)$ and $\Gamma_{4}$ the section passing through the critical point $z_{3}$ of $\phi_{4}(x)$. Then the curve $C \subset \widetilde{M}_{0, n}$ induced by $R_{\phi_{4}, \Gamma_{4}}$ satisfies $\mathscr{B}_{C}=\left\{B_{i}, B_{j}, B_{i+j}\right\}$ by (4-1).

Proof of Theorem 1.6. It follows directly from Theorem 3.2 and Theorem 4.3.

\section{Curves from birational geometry of moduli spaces}

Let $\bar{M}_{0, n}$ be the moduli space of stable $n$-pointed rational curves. The space $\bar{M}_{0, n}$ has a natural $S_{n}$ action by reordering the marked points. Let $\rho: \bar{M}_{0, n} \rightarrow \widetilde{\mathcal{M}}_{0, n}$ be the finite quotient morphism via $S_{n}$.

A weight datum $\mathscr{A}=\left(a_{1}, \ldots, a_{n}\right)$ is a sequence of rational numbers such that $0<a_{i} \leq 1$. Given a weight datum $\mathscr{A}$ satisfying $2 g-2+\sum_{i=1}^{n} a_{i}>0$, an $n$-pointed curve $\left(C ; p_{1}, \ldots, p_{n}\right)$ of genus $g$ is $\mathscr{A}$-stable if

(1) $C$ has, at worst, ordinary double points as singularities, and $p_{1}, \ldots, p_{n}$ are smooth points of $C$;

(2) $\omega_{C}\left(\sum_{i=1}^{n} a_{i} p_{i}\right)$ is ample;

(3) $\operatorname{mult}_{x}\left(\sum_{i=1}^{n} a_{i} p_{i}\right) \leq 1$ for any $x \in C$.

For any weight datum $\mathscr{A}$ such that $2 g-2+\sum_{i=1}^{n} a_{i}>0$, there exists a projective coarse moduli space $\bar{M}_{g, \mathscr{A}}$ [Hassett 2003, Theorem 2.1] of weighted $n$-pointed $\mathscr{A}$-stable curves. Note that $\bar{M}_{g, \mathscr{A}}=\bar{M}_{g, n}$ when $a_{1}=\cdots=a_{n}=1$. 
Let $\mathscr{A}_{1}=\left(a_{1}, \ldots, a_{n}\right)$ and $\mathscr{A}_{2}=\left(b_{1}, \ldots, b_{n}\right)$ be two weight data and suppose that $a_{i} \geq b_{i}$ for all $i=1,2, \ldots, n$. Then there exists a reduction morphism [Hassett 2003, Theorem 4.1]

$$
\varphi_{\mathscr{A}_{1}, \mathscr{A}_{2}}: \bar{M}_{0, \mathscr{A}_{1}} \rightarrow \bar{M}_{0, \mathscr{A}_{2}}
$$

If $\left(C, p_{1}, \ldots, p_{n}\right) \in \bar{M}_{0, \mathscr{A}_{1}}$, then $\varphi_{\mathscr{A}_{1}, \mathscr{A}_{2}}\left(C, p_{1}, \ldots, p_{n}\right)$ is obtained by collapsing components of $C$ on which $\omega_{C}+\sum b_{i} p_{i}$ fails to be ample.

Theorem 5.1. Let $\mathscr{B}^{\prime}=\left\{B_{2}, B_{k+1}, B_{k+2}, \ldots, B_{[n / 2]}\right\}$ for $2 \leq k \leq[n / 2]$; then there exists a curve $C \subset \widetilde{M}_{0, n}$ such that $\mathscr{B}_{C}=\mathscr{B}^{\prime}$.

Proof. If $k=[n / 2]$, then $\mathscr{B}^{\prime}=\left\{B_{2}\right\}$, and existence was proved in Theorem 3.1(1).

If $k=2$, then $\mathscr{B}^{\prime}=\mathscr{B}=\left\{B_{2}, B_{3}, \ldots, B_{[n / 2]}\right\}$. Taking the intersection of $n-4$ general very ample divisors of $\widetilde{M}_{0, n}$, we obtain a curve $C$ in $\widetilde{M}_{0, n}$ that intersects all the boundary divisors; that is, $\mathscr{B}_{C}=\mathscr{B}_{\text {. }}$

In the following, we assume that $3 \leq k \leq[n / 2]-1$. Let $\mathscr{A}(k)=\{1 / k, \ldots, 1 / k\}$ be the symmetric weight datum that assigns $1 / k$ to each marked point. Then $\sum_{i=1}^{n} a_{i}=n \cdot(1 / k)>2$. Let $\widetilde{M}_{0, \mathscr{A}(k)}$ be the quotient of the weighted moduli space $\bar{M}_{0, \mathscr{A}(k)}$ via the natural $S_{n}$ action. Denote by $f_{k}: \widetilde{M}_{0, n} \rightarrow \widetilde{M}_{0, \mathscr{A}(k)}$ the corresponding reduction morphism. Since three points on $\mathbb{P}^{1}$ (the two marked points and the attaching node) have no nontrivial moduli, $f_{k}$ does not contract $B_{2}$. The degree of the total weight on a rational tail with $j(3 \leq j \leq k)$ marked points is $(1 / k) \cdot j+1 \leq$ $2=-\operatorname{deg} K_{\mathbb{P}^{1}}$, where the +1 comes from the attaching node. Hence it violates the stability condition. So $f_{k}$ contracts the boundary divisors $B_{j}$ for $3 \leq j \leq k$. Furthermore, we see that $f_{k}$ contracts only these boundary divisors. Now taking the intersection of $n-4$ general very ample divisors, we obtain a curve $C$ in $\widetilde{M}_{0, A(k)}$ that is disjoint with $f_{k}\left(B_{j}\right)$ for $3 \leq j \leq k$ and that intersects all the other (not contracted) boundary divisors $B_{2}, B_{k+1}, \ldots, B_{[n / 2]}$ in the loci where $f_{k}$ is an isomorphism. Then the proper transform $C^{\prime}$ of $C$ in $\bar{M}_{0, n}$ satisfies $\mathscr{B}_{C^{\prime}}=\mathscr{B}^{\prime}=$ $\left\{B_{2}, B_{k+1}, \ldots, B_{[n / 2]}\right\}$.

Proof of Theorem 1.7. It follows from Theorems 3.1 and 5.1 and Corollary 4.2.

\section{Proof of Theorem 1.3}

Let us first introduce some notation. Below, $\left\{i_{1}, \ldots, i_{k}\right\}$ stands for $\left\{B_{i_{1}}, \ldots, B_{i_{k}}\right\}$. If $\mathscr{B}^{\prime}=\left\{i_{1}, \ldots, i_{k}\right\}$ is one of the sets in Theorem 1.6, we call $\mathscr{B}^{\prime}$ of type $T_{i_{1}, \ldots, i_{k}}$. For example, if $\mathscr{B}^{\prime}=\{2,3,5\}$, we call $\mathscr{B}^{\prime}$ of type $T_{2,3,5}$. If $\mathscr{R}^{\prime}=\left\{2, i_{1}, \ldots, i_{k}\right\}$ is one of the sets in Theorem 1.7(1)-(2), we call $\mathscr{B}^{\prime}$ of type $T_{2, *}$. If $\mathscr{B}^{\prime}=\left\{3, i_{1}, \ldots, i_{k}\right\}$ is one of the sets in Theorem 1.7(3), we call $\mathscr{B}^{\prime}$ of type $T_{3, *}$.

Proof of Theorem 1.3. For $n \leq 11$, it is easy to check that the result follows directly from Theorem 1.6 and Theorem 1.7. In the following we always assume that $12 \leq n \leq 17$. Then we have the following three cases. 
Case 1. If $\mathscr{B}^{\prime}$ is one of the sets in Theorems 1.6 and 1.7, we are done.

Case 2. If $n=14$ and $\mathscr{R}^{\prime}=\{4,7\}$, then there exists a rational function $\phi_{1}(x)$ of type $(13 ; 3,3,6,12)$ by Corollary $2.2(1)$. Denote by $\Gamma_{1}$ the section passing through the critical point $z_{4}$ of $\phi_{1}(x)$, where the multiplicity of $z_{4}$ is 12 . Then the curve $C_{1}$ in $\widetilde{M}_{0,14}$ corresponding to $R_{\phi_{1}, \Gamma_{1}}$ satisfies $\mathscr{B}_{C_{1}}=\{4,7\}$ by (4-1).

If $n=17$ and $\mathscr{B}^{\prime}=\{4,7\}$, then there exists a rational function $\phi_{2}(x)$ of type $(16 ; 3,6,6,15)$ by Corollary $2.2(1)$. Denote by $\Gamma_{2}(x)$ the section passing through the critical point $z_{4}$ of $\phi_{2}(x)$, where the multiplicity of $z_{4}$ is 15 . Then the curve $C_{2}$ in $\widetilde{M}_{0,17}$ corresponding to $R_{\phi_{2}, \Gamma_{2}}$ satisfies $\mathscr{B}_{C_{2}}=\{4,7\}$ by (4-1).

Case 3. We discuss the remaining $\mathscr{B}^{\prime}$ case by case using the method in Section 3.

Let $\mathscr{B}^{\prime}=\left\{i_{1}, \ldots, i_{k}\right\}$ be a set of boundary divisors of $\widetilde{M}_{0, n}$. If there is a sequence $\left(m_{1}, \ldots, m_{l}\right)$ satisfying

(i) $m_{1}+\cdots+m_{l}=2 n-2$,

(ii) for any $1 \leq t \leq l, m_{t} \in\left\{i_{1}-1, \ldots, i_{k}-1, n-1-i_{1}, \ldots, n-1-i_{k}, n-1, n-2\right\}$,

(iii) for any $1 \leq j \leq k$, there is $1 \leq t_{j} \leq l$ such that $i_{j}=m_{t_{j}}+1$ or $n-1-m_{t_{j}}$,

(iv) the right side of (2-2) is positive,

then there exists a rational function of type $\left(n ; m_{1}, \ldots, m_{l}\right)$ by Theorem 2.1. Thus we get a curve $C$ in $\widetilde{M}_{0, n}$ such that $\mathscr{B}_{C}=\mathscr{B}^{\prime}$ by (3-1). So we finish the proof of the theorem by giving a sequence $\left(m_{1}, \ldots, m_{l}\right)$ satisfying (i)-(iv) for each $n$ and $\mathscr{B}^{\prime}$; see Table 1-Table 10.

We now show the meaning of these tables. If $\mathscr{B}^{\prime}$ is in Case 1, we give its type in the tables. If $\mathscr{B}^{\prime}$ is in Case 3 , we give a sequence $\left(m_{1}, \ldots, m_{l}\right)$ satisfying (i)-(iv) in the tables. For example, if $\mathscr{B}^{\prime}=\{2,3\}$, then $\left(m_{1}, \ldots, m_{l}\right)$ is $T_{2, *}$ in Table 1 ; by

\begin{tabular}{|cc|cc|cc|}
\hline $\mathscr{R}^{\prime}$ & $\left(m_{1}, \ldots, m_{l}\right)$ & $\mathscr{B}^{\prime}$ & $\left(m_{1}, \ldots, m_{l}\right)$ & $\mathscr{B}^{\prime}$ & $\left(m_{1}, \ldots, m_{l}\right)$ \\
\hline$\{2,3\}$ & $T_{2, *}$ & $\{2,4\}$ & $T_{2, *}$ & $\{2,5\}$ & $T_{2, *}$ \\
$\{2,6\}$ & $T_{2, *}$ & $\{3,4\}$ & $T_{3, *}$ & $\{3,5\}$ & $T_{3, *}$ \\
$\{3,6\}$ & $T_{3, *}$ & $\{4,5\}$ & $T_{4,5}$ & $\{4,6\}$ & $T_{4,6}$ \\
$\{5,6\}$ & $T_{5,6}$ & & & & \\
\hline$\{2,3,4\}$ & $T_{2, *}$ & $\{2,3,5\}$ & $T_{2, *}$ & $\{2,3,6\}$ & $T_{2, *}$ \\
$\{2,4,5\}$ & $T_{2, *}$ & $\{2,4,6\}$ & $T_{2, *}$ & $\{2,5,6\}$ & $T_{2, *}$ \\
$\{3,4,5\}$ & $T_{3,4,5}$ & $\{3,4,6\}$ & $T_{3,4,6}$ & $\{3,5,6\}$ & $T_{3,5,6}$ \\
$\{4,5,6\}$ & $(4,5,6,7)$ & & & & \\
\hline$\{2,3,4,5\}$ & $T_{2, *}$ & $\{2,3,4,6\}$ & $T_{2, *}$ & $\{2,3,5,6\}$ & $(2,5,6,9)$ \\
$\{2,4,5,6\}$ & $T_{2, *}$ & $\{3,4,5,6\}$ & $(3,5,6,8)$ & & \\
\hline
\end{tabular}

Table 1. $\tilde{M}_{0,12}$. 


\begin{tabular}{|cc|cc|cc|}
\hline $\mathscr{B}^{\prime}$ & $\left(m_{1}, \ldots, m_{l}\right)$ & $\mathscr{B}^{\prime}$ & $\left(m_{1}, \ldots, m_{l}\right)$ & $\mathscr{B}^{\prime}$ & $\left(m_{1}, \ldots, m_{l}\right)$ \\
\hline$\{2,3\}$ & $T_{2, *}$ & $\{2,4\}$ & $T_{2, *}$ & $\{2,5\}$ & $T_{2, *}$ \\
$\{2,6\}$ & $T_{2, *}$ & $\{3,4\}$ & $T_{3, *}$ & $\{3,5\}$ & $T_{3, *}$ \\
$\{3,6\}$ & $T_{3, *}$ & $\{4,5\}$ & $T_{4,5}$ & $\{4,6\}$ & $T_{4,6}$ \\
$\{5,6\}$ & $T_{5,6}$ & & & & \\
\hline$\{2,3,4\}$ & $T_{2, *}$ & $\{2,3,5\}$ & $T_{2, *}$ & $\{2,3,6\}$ & $T_{2, *}$ \\
$\{2,4,5\}$ & $T_{2, *}$ & $\{2,4,6\}$ & $T_{2, *}$ & $\{2,5,6\}$ & $T_{2, *}$ \\
$\{3,4,5\}$ & $T_{3,4,5}$ & $\{3,4,6\}$ & $T_{3,4,6}$ & $\{3,5,6\}$ & $T_{3,5,6}$ \\
$\{4,5,6\}$ & $(3,6,7,8)$ & & & & \\
\hline$\{2,3,4,5\}$ & $T_{2, *}$ & $\{2,3,4,6\}$ & $T_{2, *}$ & $\{2,3,5,6\}$ & $T_{2, *}$ \\
$\{2,4,5,6\}$ & $T_{2, *}$ & $\{3,4,5,6\}$ & $(2,3,4,6,9)$ & & \\
\hline
\end{tabular}

Table 2. $\widetilde{M}_{0,13}$.

this we mean that there is a curve $C$ in $\widetilde{M}_{0,12}$ of type $T_{2, *}$ with $\mathscr{B}_{C}=\left\{B_{2}, B_{3}\right\}$. If $\mathscr{B}^{\prime}=\{4,5,6\}$, then $\left(m_{1}, \ldots, m_{l}\right)=(4,5,6,7)$ in Table 1 ; by this we mean that there exists a curve $C$ in $\widetilde{M}_{0,12}$ with $\mathscr{B}_{C}=\{4,5,6\}$, where $C$ is induced by a rational function of type $(12 ; 4,5,6,7)$.

Note that only the cases of $\mathscr{B}^{\prime}$ with $\left|\mathscr{B}^{\prime}\right|=1$ and $\left|\mathscr{B}^{\prime}\right|=[n / 2]-1$ are not contained in these tables, since the theorem for such $\mathscr{B}^{\prime}$ holds true for any $n$; see Theorem 4.3(1) and Theorem 5.1 for $k=2$.

Remark 6.1. Assume that $n=18$ and $\mathscr{B}^{\prime}=\left\{B_{3}, B_{4}, \ldots, B_{9}\right\}$. Suppose that there exists a sequence $\left(m_{1}, \ldots, m_{l}\right)$ satisfying (i)-(iv) for $\mathscr{S}^{\prime}$. From (ii) and (iii), we know that

$$
m_{1}+\cdots+m_{l} \geq \sum_{i=3}^{9}(i-1)=35>2 n-2=34,
$$

which contradicts (i). Hence the method in Section 3 is invalid for $\widetilde{M}_{0,18}$. Similarly, we know that the method in Section 4 is also invalid.

\begin{tabular}{|cc|cc|cc|}
\hline $\mathscr{B}^{\prime}$ & $\left(m_{1}, \ldots, m_{l}\right)$ & $\mathscr{B}^{\prime}$ & $\left(m_{1}, \ldots, m_{l}\right)$ & $\mathscr{B}^{\prime}$ & $\left(m_{1}, \ldots, m_{l}\right)$ \\
\hline$\{2,3\}$ & $T_{2, *}$ & $\{2,4\}$ & $T_{2, *}$ & $\{2,5\}$ & $T_{2, *}$ \\
$\{2,6\}$ & $T_{2, *}$ & $\{2,7\}$ & $T_{2, *}$ & $\{3,4\}$ & $T_{3, *}$ \\
$\{3,5\}$ & $T_{3, *}$ & $\{3,6\}$ & $T_{3, *}$ & $\{3,7\}$ & $T_{3, *}$ \\
$\{4,5\}$ & $T_{4,5}$ & $\{4,6\}$ & $T_{4,6}$ & $\{4,7\}$ & see Case 2 \\
$\{5,6\}$ & $T_{5,6}$ & $\{5,7\}$ & $T_{5,7}$ & $\{6,7\}$ & $T_{6,7}$ \\
\hline
\end{tabular}

Table 3. $\widetilde{M}_{0,14}$ for $\left|\mathscr{B}^{\prime}\right|=2$. 


\begin{tabular}{|cc|cc|cc|}
\hline $\mathscr{B}^{\prime}$ & $\left(m_{1}, \ldots, m_{l}\right)$ & $\mathscr{B}^{\prime}$ & $\left(m_{1}, \ldots, m_{l}\right)$ & $\mathscr{R}^{\prime}$ & $\left(m_{1}, \ldots, m_{l}\right)$ \\
\hline$\{2,3,4\}$ & $T_{2, *}$ & $\{2,3,5\}$ & $T_{2, *}$ & $\{2,3,6\}$ & $T_{2, *}$ \\
$\{2,3,7\}$ & $T_{2, *}$ & $\{2,4,5\}$ & $T_{2, *}$ & $\{2,4,6\}$ & $T_{2, *}$ \\
$\{2,4,7\}$ & $T_{2, *}$ & $\{2,5,6\}$ & $T_{2, *}$ & $\{2,5,7\}$ & $T_{2, *}$ \\
$\{2,6,7\}$ & $T_{2, *}$ & $\{3,4,5\}$ & $T_{3,4,5}$ & $\{3,4,6\}$ & $T_{3,4,6}$ \\
$\{3,4,7\}$ & $T_{3,4,7}$ & $\{3,5,6\}$ & $T_{3,5,6}$ & $\{3,5,7\}$ & $T_{3,5,7}$ \\
$\{3,6,7\}$ & $T_{3,6,7}$ & $\{4,5,6\}$ & $(4,5,8,9)$ & $\{4,5,7\}$ & $T_{4,5,7}$ \\
$\{4,6,7\}$ & $(3,3,5,6,9)$ & $\{5,6,7\}$ & $(5,6,7,8)$ & & $(1,5,5,6,9)$ \\
\hline$\{2,3,4,5\}$ & $T_{2, *}$ & $\{2,3,4,6\}$ & $T_{2, *}$ & $\{2,3,4,7\}$ & $T_{2, *}$ \\
$\{2,3,5,6\}$ & $T_{2, *}$ & $\{2,3,5,7\}$ & $T_{2, *}$ & $\{2,3,6,7\}$ & $(1,2,6,7,10)$ \\
$\{2,4,5,6\}$ & $T_{2, *}$ & $\{2,4,5,7\}$ & $(1,4,6,6,9)$ & $\{2,4,6,7\}$ & $(1,5,5,6,10)$ \\
$\{2,5,6,7\}$ & $T_{2, *}$ & $\{3,4,5,6\}$ & $(3,5,8,10)$ & $\{3,4,5,7\}$ & $(3,3,4,6,10)$ \\
$\{3,4,6,7\}$ & $(3,6,7,10)$ & $\{3,5,6,7\}$ & $(2,5,5,6,8)$ & $\{4,5,6,7\}$ & $(4,6,7,9)$ \\
\hline \multicolumn{7}{|c|}{$\left(m_{1}, \ldots, m_{l}\right)$} & $\mathscr{B}^{\prime}$ & $\left(m_{1}, \ldots, m_{l}\right)$ \\
\hline
\end{tabular}

Table 4. $\widetilde{M}_{0,14}$ for $3 \leq\left|\mathscr{B}^{\prime}\right| \leq 5$.

\begin{tabular}{|cc|cc|cc|}
\hline $\mathscr{B}^{\prime}$ & $\left(m_{1}, \ldots, m_{l}\right)$ & $\mathscr{B}^{\prime}$ & $\left(m_{1}, \ldots, m_{l}\right)$ & $\mathscr{B}^{\prime}$ & $\left(m_{1}, \ldots, m_{l}\right)$ \\
\hline$\{2,3\}$ & $T_{2, *}$ & $\{2,4\}$ & $T_{2, *}$ & $\{2,5\}$ & $T_{2, *}$ \\
$\{2,6\}$ & $T_{2, *}$ & $\{2,7\}$ & $T_{2, *}$ & $\{3,4\}$ & $T_{3, *}$ \\
$\{3,5\}$ & $T_{3, *}$ & $\{3,6\}$ & $T_{3, *}$ & $\{3,7\}$ & $T_{3, *}$ \\
$\{4,5\}$ & $T_{4,5}$ & $\{4,6\}$ & $T_{4,6}$ & $\{4,7\}$ & $(6,6,6,10)$ \\
$\{5,6\}$ & $T_{5,6}$ & $\{5,7\}$ & $T_{5,7}$ & $\{6,7\}$ & $T_{6,7}$ \\
\hline$\{2,3,4\}$ & $T_{2, *}$ & $\{2,3,5\}$ & $T_{2, *}$ & $\{2,3,6\}$ & $T_{2, *}$ \\
$\{2,3,7\}$ & $T_{2, *}$ & $\{2,4,5\}$ & $T_{2, *}$ & $\{2,4,6\}$ & $T_{2, *}$ \\
$\{2,4,7\}$ & $T_{2, *}$ & $\{2,5,6\}$ & $T_{2, *}$ & $\{2,5,7\}$ & $T_{2, *}$ \\
$\{2,6,7\}$ & $T_{2, *}$ & $\{3,4,5\}$ & $T_{3,4,5}$ & $\{3,4,6\}$ & $T_{3,4,6}$ \\
$\{3,4,7\}$ & $T_{3,4,7}$ & $\{3,5,6\}$ & $T_{3,5,6}$ & $\{3,5,7\}$ & $T_{3,5,7}$ \\
$\{3,6,7\}$ & $T_{3,6,7}$ & $\{4,5,6\}$ & $(4,5,9,10)$ & $\{4,5,7\}$ & $T_{4,5,7}$ \\
$\{4,6,7\}$ & $(3,7,8,10)$ & $\{5,6,7\}$ & $(4,7,8,9)$ & & \\
\hline$\{2,3,4,5\}$ & $T_{2, *}$ & $\{2,3,4,6\}$ & $T_{2, *}$ & $\{2,3,4,7\}$ & $T_{2, *}$ \\
$\{2,3,5,6\}$ & $T_{2, *}$ & $\{2,3,5,7\}$ & $T_{2, *}$ & $\{2,3,6,7\}$ & $T_{2, *}$ \\
$\{2,4,5,6\}$ & $T_{2, *}$ & $\{2,4,5,7\}$ & $T_{2, *}$ & $\{2,4,6,7\}$ & $(1,3,5,7,12)$ \\
$\{2,5,6,7\}$ & $T_{2, *}$ & $\{3,4,5,6\}$ & $T_{3, *}$ & $\{3,4,5,7\}$ & $(2,7,9,10)$ \\
$\{3,4,6,7\}$ & $(3,6,8,11)$ & $\{3,5,6,7\}$ & $(2,4,5,6,11)$ & $\{4,5,6,7\}$ & $(3,4,5,6,10)$ \\
\hline
\end{tabular}

Table 5. $\widetilde{M}_{0,15}$ for $2 \leq\left|\mathscr{B}^{\prime}\right| \leq 4$. 


\begin{tabular}{|cc|cc|}
\hline $\mathscr{B}^{\prime}$ & $\left(m_{1}, \ldots, m_{l}\right)$ & $\mathscr{B}^{\prime}$ & $\left(m_{1}, \ldots, m_{l}\right)$ \\
\hline$\{2,3,4,5,6\}$ & $(1,2,3,4,8,10)$ & $\{2,3,4,5,7\}$ & $(2,3,4,7,12)$ \\
$\{2,3,4,6,7\}$ & $(2,3,5,6,12)$ & $\{2,3,5,6,7\}$ & $(1,4,5,7,11)$ \\
$\{2,4,5,6,7\}$ & $T_{2, *}$ & $\{3,4,5,6,7\}$ & $(2,4,5,7,10)$ \\
\hline
\end{tabular}

Table 6. $\widetilde{M}_{0,15}$ for $\left|\Re^{\prime}\right|=5$.

\begin{tabular}{|c|c|c|c|c|c|}
\hline $\mathscr{B}^{\prime}$ & $\left(m_{1}, \ldots, m_{l}\right)$ & $\mathscr{H}^{\prime}$ & $\left(m_{1}, \ldots, m_{l}\right)$ & $\mathscr{H}^{\prime}$ & $\left(m_{1}, \ldots, m_{l}\right)$ \\
\hline$\{2,3\}$ & $T_{2, *}$ & $\{2,4\}$ & $T_{2, *}$ & $\{2,5\}$ & $T_{2, *}$ \\
\hline$\{2,6\}$ & $T_{2, *}$ & $\{2,7\}$ & $T_{2, *}$ & $\{2,8\}$ & $T_{2, *}$ \\
\hline$\{3,4\}$ & $T_{3, *}$ & $\{3,5\}$ & $T_{3, *}$ & $\{3,6\}$ & $T_{3, *}$ \\
\hline$\{3,7\}$ & $T_{3, *}$ & $\{3,8\}$ & $T_{3, *}$ & $\{4,5\}$ & $T_{4,5}$ \\
\hline$\{4,6\}$ & $T_{4,6}$ & $\{4,7\}$ & $(8,11,11)$ & $\{4,8\}$ & $(3,3,3,7,7,7)$ \\
\hline$\{5,6\}$ & $T_{5,6}$ & $\{5,7\}$ & $T_{5,7}$ & $\{5,8\}$ & $(4,4,4,4,7,7)$ \\
\hline$\{6,7\}$ & $T_{6,7}$ & $\{6,8\}$ & $T_{6,8}$ & $\{7,8\}$ & $T_{7,8}$ \\
\hline$\{2,3,4\}$ & $T_{2, *}$ & $\{2,3,5\}$ & $T_{2, *}$ & $\{2,3,6\}$ & $T_{2, *}$ \\
\hline$\{2,3,7\}$ & $T_{2, *}$ & $\{2,3,8\}$ & $T_{2, *}$ & $\{2,4,5\}$ & $T_{2, *}$ \\
\hline$\{2,4,6\}$ & $T_{2, *}$ & $\{2,4,7\}$ & $T_{2, *}$ & $\{2,4,8\}$ & $T_{2, *}$ \\
\hline$\{2,5,6\}$ & $T_{2, *}$ & $\{2,5,7\}$ & $T_{2, *}$ & $\{2,5,8\}$ & $T_{2, *}$ \\
\hline$\{2,6,7\}$ & $T_{2, *}$ & $\{2,6,8\}$ & $T_{2, *}$ & $\{2,7,8\}$ & $T_{2, *}$ \\
\hline$\{3,4,5\}$ & $T_{3,4,5}$ & $\{3,4,6\}$ & $T_{3,4,6}$ & $\{3,4,7\}$ & $T_{3,4,7}$ \\
\hline$\{3,4,8\}$ & $T_{3,4,8}$ & $\{3,5,6\}$ & $T_{3,5,6}$ & $\{3,5,7\}$ & $T_{3,5,7}$ \\
\hline$\{3,5,8\}$ & $T_{3,5,8}$ & $\{3,6,7\}$ & $T_{3,6,7}$ & $\{3,6,8\}$ & $T_{3,6,8}$ \\
\hline$\{3,7,8\}$ & $T_{3,7,8}$ & $\{4,5,6\}$ & $(9,10,11)$ & $\{4,5,7\}$ & $T_{4,5,7}$ \\
\hline$\{4,5,8\}$ & $T_{4,5,8}$ & $\{4,6,7\}$ & $(5,6,8,11)$ & $\{4,6,8\}$ & $T_{4,6,8}$ \\
\hline$\{4,7,8\}$ & $(6,6,7,11)$ & $\{5,6,7\}$ & $(5,6,9,10)$ & $\{5,6,8\}$ & $(4,7,9,10)$ \\
\hline$\{5,7,8\}$ & $(6,7,7,10)$ & $\{6,7,8\}$ & $(6,7,8,9)$ & & \\
\hline$\{2,3,4,5\}$ & $T_{2, *}$ & $\{2,3,4,6\}$ & $T_{2, *}$ & $\{2,3,4,7\}$ & $T_{2, *}$ \\
\hline$\{2,3,4,8\}$ & $T_{2, *}$ & $\{2,3,5,6\}$ & $T_{2, *}$ & $\{2,3,5,7\}$ & $T_{2, *}$ \\
\hline$\{2,3,5,8\}$ & $T_{2, *}$ & $\{2,3,6,7\}$ & $T_{2, *}$ & $\{2,3,6,8\}$ & $T_{2, *}$ \\
\hline$\{2,3,7,8\}$ & $(2,7,8,13)$ & $\{2,4,5,6\}$ & $T_{2, *}$ & $\{2,4,5,7\}$ & $T_{2, *}$ \\
\hline$\{2,4,5,8\}$ & $T_{2, *}$ & $\{2,4,6,7\}$ & $T_{2, *}$ & $\{2,4,6,8\}$ & $(1,3,5,5,7,9)$ \\
\hline$\{2,4,7,8\}$ & $(1,3,6,7,13)$ & $\{2,5,6,7\}$ & $(1,4,6,9,10)$ & $\{2,5,6,8\}$ & $(1,4,5,7,13)$ \\
\hline$\{2,5,7,8\}$ & $(4,6,7,13)$ & $\{2,6,7,8\}$ & $T_{2, *}$ & $\{3,4,5,6\}$ & $(2,3,4,9,12)$ \\
\hline$\{3,4,5,7\}$ & $(2,2,3,4,8,11)$ & $\{3,4,5,8\}$ & $(2,7,10,11)$ & $\{3,4,6,7\}$ & $(2,8,9,11)$ \\
\hline$\{3,4,6,8\}$ & $(3,3,5,7,12)$ & $\{3,4,7,8\}$ & $(2,2,7,8,11)$ & $\{3,5,6,7\}$ & $(2,2,4,5,8,9)$ \\
\hline$\{3,5,6,8\}$ & $(2,2,7,9,10)$ & $\{3,5,7,8\}$ & $(2,2,4,7,7,8)$ & $\{3,6,7,8\}$ & $(5,6,7,12)$ \\
\hline$\{4,5,6,7\}$ & $(4,6,9,11)$ & $\{4,5,6,8\}$ & $(3,4,5,7,11)$ & $\{4,5,7,8\}$ & $(4,7,8,11)$ \\
\hline$\{4,6,7,8\}$ & $(3,5,6,7,9)$ & $\{5,6,7,8\}$ & $(5,7,8,10)$ & & \\
\hline
\end{tabular}

Table 7. $\widetilde{M}_{0,16}$ for $2 \leq\left|\mathscr{B}^{\prime}\right| \leq 4$. 


\begin{tabular}{|cc|cc|}
\hline $\mathscr{B}^{\prime}$ & $\left(m_{1}, \ldots, m_{l}\right)$ & $\mathscr{B}^{\prime}$ & $\left(m_{1}, \ldots, m_{l}\right)$ \\
\hline$\{2,3,4,5,6\}$ & $(1,2,3,5,9,10)$ & $\{2,3,4,5,7\}$ & $(1,2,3,6,8,10)$ \\
$\{2,3,4,5,8\}$ & $(1,2,3,4,7,13)$ & $\{2,3,4,6,7\}$ & $(1,2,3,5,6,13)$ \\
$\{2,3,4,6,8\}$ & $(2,3,5,7,13)$ & $\{2,3,4,7,8\}$ & $(1,1,3,6,7,12)$ \\
$\{2,3,5,6,7\}$ & $(1,2,4,5,6,12)$ & $\{2,3,5,6,8\}$ & $(1,1,4,5,7,12)$ \\
$\{2,3,5,7,8\}$ & $(1,4,6,7,12)$ & $\{2,3,6,7,8\}$ & $(1,2,5,6,7,9)$ \\
$\{2,4,5,6,7\}$ & $(1,3,4,5,6,11)$ & $\{2,4,5,6,8\}$ & $(1,3,4,5,7,10)$ \\
$\{2,4,5,7,8\}$ & $(1,3,4,6,7,9)$ & $\{2,4,6,7,8\}$ & $(1,3,5,6,7,8)$ \\
$\{2,5,6,7,8\}$ & $T_{2, *}$ & $\{3,4,5,6,7\}$ & $(2,3,4,5,6,10)$ \\
$\{3,4,5,6,8\}$ & $(2,3,4,5,7,9)$ & $\{3,4,5,7,8\}$ & $(2,2,4,5,6,11)$ \\
$\{3,4,6,7,8\}$ & $(2,3,5,6,7,7)$ & $\{3,5,6,7,8\}$ & $(2,5,6,7,10)$ \\
$\{4,5,6,7,8\}$ & $(3,4,5,5,6,7)$ & & \\
\hline$\{2,3,4,5,6,7\}$ & $(1,2,3,4,5,6,9)$ & $\{2,3,4,5,6,8\}$ & $(1,2,4,5,7,11)$ \\
$\{2,3,4,5,7,8\}$ & $(1,1,2,3,6,7,10)$ & $\{2,3,4,6,7,8\}$ & $(1,2,3,7,8,9)$ \\
$\{2,3,5,6,7,8\}$ & $(1,2,4,5,5,6,7)$ & $\{2,4,5,6,7,8\}$ & $T_{2, *}$ \\
$\{3,4,5,6,7,8\}$ & $(2,3,3,4,5,6,7)$ & & \\
\hline
\end{tabular}

Table 8. $\widetilde{M}_{0,16}$ for $5 \leq\left|\mathscr{B}^{\prime}\right| \leq 6$.

\begin{tabular}{|cc|cc|cc|}
\hline $\mathscr{B}^{\prime}$ & $\left(m_{1}, \ldots, m_{l}\right)$ & $\mathscr{B}^{\prime}$ & $\left(m_{1}, \ldots, m_{l}\right)$ & $\mathscr{B}^{\prime}$ & $\left(m_{1}, \ldots, m_{l}\right)$ \\
\hline$\{2,3\}$ & $T_{2, *}$ & $\{2,4\}$ & $T_{2, *}$ & $\{2,5\}$ & $T_{2, *}$ \\
$\{2,6\}$ & $T_{2, *}$ & $\{2,7\}$ & $T_{2, *}$ & $\{2,8\}$ & $T_{2, *}$ \\
$\{3,4\}$ & $T_{3, *}$ & $\{3,5\}$ & $T_{3, *}$ & $\{3,6\}$ & $T_{3, *}$ \\
$\{3,7\}$ & $T_{3, *}$ & $\{3,8\}$ & $T_{3, *}$ & $\{4,5\}$ & $T_{4,5}$ \\
$\{4,6\}$ & $T_{4,6}$ & $\{4,7\}$ & see Case 2 & $\{4,8\}$ & $(3,3,7,7,12)$ \\
$\{5,6\}$ & $T_{5,6}$ & $\{5,7\}$ & $T_{5,7}$ & $\{5,8\}$ & $(7,7,7,11)$ \\
$\{6,7\}$ & $T_{6,7}$ & $\{6,8\}$ & $T_{6,8}$ & $\{7,8\}$ & $T_{7,8}$ \\
\hline$\{2,3,4\}$ & $T_{2, *}$ & $\{2,3,5\}$ & $T_{2, *}$ & $\{2,3,6\}$ & $T_{2, *}$ \\
$\{2,3,7\}$ & $T_{2, *}$ & $\{2,3,8\}$ & $T_{2, *}$ & $\{2,4,5\}$ & $T_{2, *}$ \\
$\{2,4,6\}$ & $T_{2, *}$ & $\{2,4,7\}$ & $T_{2, *}$ & $\{2,4,8\}$ & $T_{2, *}$ \\
$\{2,5,6\}$ & $T_{2, *}$ & $\{2,5,7\}$ & $T_{2, *}$ & $\{2,5,8\}$ & $T_{2, *}$ \\
$\{2,6,7\}$ & $T_{2, *}$ & $\{2,6,8\}$ & $T_{2, *}$ & $\{2,7,8\}$ & $T_{2, *}$ \\
$\{3,4,5\}$ & $T_{3,4,5}$ & $\{3,4,6\}$ & $T_{3,4,6}$ & $\{3,4,7\}$ & $T_{3,4,7}$ \\
$\{3,4,8\}$ & $T_{3,4,8}$ & $\{3,5,6\}$ & $T_{3,5,6}$ & $\{3,5,7\}$ & $T_{3,5,7}$ \\
$\{3,5,8\}$ & $T_{3,5,8}$ & $\{3,6,7\}$ & $T_{3,6,7}$ & $\{3,6,8\}$ & $T_{3,6,8}$ \\
$\{3,7,8\}$ & $T_{3,7,8}$ & $\{4,5,6\}$ & $(4,5,11,12)$ & $\{4,5,7\}$ & $T_{4,5,7}$ \\
$\{4,5,8\}$ & $T_{4,5,8}$ & $\{4,6,7\}$ & $(5,6,9,12)$ & $\{4,6,8\}$ & $T_{4,6,8}$ \\
$\{4,7,8\}$ & $(6,6,8,12)$ & $\{5,6,7\}$ & $(5,6,10,11)$ & $\{5,6,8\}$ & $(4,7,10,11)$ \\
$\{5,7,8\}$ & $(6,7,8,11)$ & $\{6,7,8\}$ & $(6,7,9,10)$ & & \\
\hline
\end{tabular}

Table 9. $\widetilde{M}_{0,17}$ for $2 \leq\left|\mathscr{B}^{\prime}\right| \leq 3$. 


\begin{tabular}{|cc|cc|cc|}
\hline $\mathscr{B}^{\prime}$ & $\left(m_{1}, \ldots, m_{l}\right)$ & $\mathscr{B}^{\prime}$ & $\left(m_{1}, \ldots, m_{l}\right)$ & $\mathscr{B}^{\prime}$ & $\left(m_{1}, \ldots, m_{l}\right)$ \\
\hline$\{2,3,4,5\}$ & $T_{2, *}$ & $\{2,3,4,6\}$ & $T_{2, *}$ & $\{2,3,4,7\}$ & $T_{2, *}$ \\
$\{2,3,4,8\}$ & $T_{2, *}$ & $\{2,3,5,6\}$ & $T_{2, *}$ & $\{2,3,5,7\}$ & $T_{2, *}$ \\
$\{2,3,5,8\}$ & $T_{2, *}$ & $\{2,3,6,7\}$ & $T_{2, *}$ & $\{2,3,6,8\}$ & $T_{2, *}$ \\
$\{2,3,7,8\}$ & $T_{2, *}$ & $\{2,4,5,6\}$ & $T_{2, *}$ & $\{2,4,5,7\}$ & $T_{2, *}$ \\
$\{2,4,5,8\}$ & $T_{2, *}$ & $\{2,4,6,7\}$ & $T_{2, *}$ & $\{2,4,6,8\}$ & $T_{2, *}$ \\
$\{2,4,7,8\}$ & $(1,3,7,9,12)$ & $\{2,5,6,7\}$ & $T_{2, *}$ & $\{2,5,6,8\}$ & $(1,5,7,8,11)$ \\
$\{2,5,7,8\}$ & $(4,6,8,14)$ & $\{2,6,7,8\}$ & $T_{2, *}$ & $\{3,4,5,6\}$ & $T_{3, *}$ \\
$\{3,4,5,7\}$ & $T_{3, *}$ & $\{3,4,5,8\}$ & $T_{3, *}$ & $\{3,4,6,7\}$ & $T_{3, *}$ \\
$\{3,4,6,8\}$ & $(2,8,10,12)$ & $\{3,4,7,8\}$ & $(3,7,9,13)$ & $\{3,5,6,7\}$ & $(2,9,10,11)$ \\
$\{3,5,6,8\}$ & $(2,4,5,8,13)$ & $\{3,5,7,8\}$ & $(2,4,6,7,13)$ & $\{3,6,7,8\}$ & $(5,6,8,13)$ \\
$\{4,5,6,7\}$ & $(4,6,10,12)$ & $\{4,5,6,8\}$ & $(3,4,5,8,12)$ & $\{4,5,7,8\}$ & $(4,7,9,12)$ \\
$\{4,6,7,8\}$ & $(3,6,6,7,10)$ & $\{5,6,7,8\}$ & $(5,7,9,11)$ & & \\
\hline
\end{tabular}

\begin{tabular}{|cc|cc|}
\hline $\mathscr{B}^{\prime}$ & $\left(m_{1}, \ldots, m_{l}\right)$ & $\mathscr{B}^{\prime}$ & $\left(m_{1}, \ldots, m_{l}\right)$ \\
\hline$\{2,3,4,5,6\}$ & $T_{2, *}$ & $\{2,3,4,5,7\}$ & $T_{2, *}$ \\
$\{2,3,4,5,8\}$ & $(1,2,3,4,8,14)$ & $\{2,3,4,6,7\}$ & $(2,2,3,5,6,14)$ \\
$\{2,3,4,6,8\}$ & $(2,3,5,8,14)$ & $\{2,3,4,7,8\}$ & $(2,3,6,7,14)$ \\
$\{2,3,5,6,7\}$ & $(1,4,5,9,13)$ & $\{2,3,5,6,8\}$ & $(2,4,5,7,14)$ \\
$\{2,3,5,7,8\}$ & $(1,4,6,8,13)$ & $\{2,3,6,7,8\}$ & $(1,5,6,7,13)$ \\
$\{2,4,5,6,7\}$ & $(3,4,5,6,14)$ & $\{2,4,5,6,8\}$ & $(1,3,7,10,11)$ \\
$\{2,4,5,7,8\}$ & $(1,3,8,9,11)$ & $\{2,4,6,7,8\}$ & $(1,5,6,8,12)$ \\
$\{2,5,6,7,8\}$ & $T_{2, *}$ & $\{3,4,5,6,7\}$ & $(2,4,5,9,12)$ \\
$\{3,4,5,6,8\}$ & $(3,4,5,7,13)$ & $\{3,4,5,7,8\}$ & $(2,3,7,9,11)$ \\
$\{3,4,6,7,8\}$ & $(2,5,6,7,12)$ & $\{3,5,6,7,8\}$ & $(2,5,6,8,11)$ \\
$\{4,5,6,7,8\}$ & $(3,5,6,7,11)$ & & \\
\hline$\{2,3,4,5,6,7\}$ & $(1,3,4,5,6,13)$ & $\{2,3,4,5,6,8\}$ & $(1,2,4,5,8,12)$ \\
$\{2,3,4,5,7,8\}$ & $(1,2,3,4,6,7,9)$ & $\{2,3,4,6,7,8\}$ & $(1,2,3,7,9,10)$ \\
$\{2,3,5,6,7,8\}$ & $(1,2,5,6,7,11)$ & $\{2,4,5,6,7,8\}$ & $T_{2, *}$ \\
$\{3,4,5,6,7,8\}$ & $(2,3,4,6,7,10)$ & & \\
\hline
\end{tabular}

Table 10. $\widetilde{M}_{0,17}$ for $4 \leq\left|\Re^{\prime}\right| \leq 6$.

\section{Acknowledgements}

I thank Fei Ye and Zhenhua Qu for discussions on the cone of moduli spaces. I am very grateful to Prof. Shengli Tan for his encouragement and lots of helpful discussions. I would like to sincerely thank Hao Sun and Yifei Chen for reading the earlier version and for their valuable comments. The result of Theorem 5.1 is due to the referee, and I thank him very much for his help. Finally, I would like to express my appreciation to the referee for pointing out how to improve the paper 
and providing many valuable suggestions in rewriting the manuscript to make the paper more readable.

\section{References}

[Gibney 2009] A. Gibney, "Numerical criteria for divisors on $\bar{M}_{g}$ to be ample", Compos. Math. 145:5 (2009), 1227-1248. MR Zbl

[Harris and Morrison 1998] J. Harris and I. Morrison, Moduli of curves, Graduate Texts in Mathematics 187, Springer, New York, 1998. MR Zbl

[Hassett 2003] B. Hassett, "Moduli spaces of weighted pointed stable curves", Adv. Math. 173:2 (2003), 316-352. MR Zbl

[Kodaira 1967] K. Kodaira, "A certain type of irregular algebraic surfaces", J. Analyse Math. 19 (1967), 207-215. MR Zbl

[Liu 2016] X.-L. Liu, "Modular invariants and singularity indices of hyperelliptic fibrations", preprint, 2016. To appear in Chin. Ann. Math. (B). arXiv

[Liu and Tan 2013] X.-L. Liu and S.-L. Tan, "Families of hyperelliptic curves with maximal slopes", Sci. China Math. 56:9 (2013), 1743-1750. MR Zbl

[Moriwaki 1998] A. Moriwaki, "Relative Bogomolov's inequality and the cone of positive divisors on the moduli space of stable curves", J. Amer. Math. Soc. 11:3 (1998), 569-600. MR Zbl

[Scherbak 2002] I. Scherbak, "Rational functions with prescribed critical points", Geom. Funct. Anal. 12:6 (2002), 1365-1380. MR Zbl

[Tan 2010] S.-L. Tan, "Chern numbers of a singular fiber, modular invariants and isotrivial families of curves", Acta Math. Vietnam. 35:1 (2010), 159-172. MR Zbl

Received December 1, 2014. Revised December 19, 2015.

XiaO-Lei LiU

ACADEmy of Mathematics and Systems Science

ChINESE ACADEMY OF SCIENCE

55, EAST ZHONGGUANCUN ROAD

HAIDIAN

BEIJING, 100190

CHINA

xlliu1124@amss.ac.cn 


\title{
PACIFIC JOURNAL OF MATHEMATICS
}

Founded in 1951 by E. F. Beckenbach (1906-1982) and F. Wolf (1904-1989)

$$
\text { msp.org/pjm }
$$

\section{EDITORS}

\author{
Don Blasius (Managing Editor) \\ Department of Mathematics \\ University of California \\ Los Angeles, CA 90095-1555 \\ blasius@math.ucla.edu
}

\author{
Paul Balmer \\ Department of Mathematics \\ University of California \\ Los Angeles, CA 90095-1555 \\ balmer@math.ucla.edu \\ Robert Finn \\ Department of Mathematics \\ Stanford University \\ Stanford, CA 94305-2125 \\ finn@math.stanford.edu \\ Sorin Popa \\ Department of Mathematics \\ University of California \\ Los Angeles, CA 90095-1555 \\ popa@math.ucla.edu
}

\author{
Vyjayanthi Chari \\ Department of Mathematics \\ University of California \\ Riverside, CA 92521-0135 \\ chari@math.ucr.edu \\ Kefeng Liu \\ Department of Mathematics \\ University of California \\ Los Angeles, CA 90095-1555 \\ liu@math.ucla.edu \\ Igor Pak \\ Department of Mathematics \\ University of California \\ Los Angeles, CA 90095-1555 \\ pak.pjm@gmail.com \\ Paul Yang \\ Department of Mathematics \\ Princeton University \\ Princeton NJ 08544-1000 \\ yang@math.princeton.edu
}

\section{PRODUCTION}

Silvio Levy, Scientific Editor, production@msp.org

\section{SUPPORTING INSTITUTIONS}

ACADEMIA SINICA, TAIPEI

CALIFORNIA INST. OF TECHNOLOGY

STANFORD UNIVERSITY

UNIV. OF BRITISH COLUMBIA

UNIV. OF CALIFORNIA, BERKELEY

UNIV. OF CALIFORNIA, DAVIS

UNIV. OF CALIFORNIA, LOS ANGELES

UNIV. OF CALIFORNIA, RIVERSIDE

UNIV. OF CALIFORNIA, SAN DIEGO

UNIV. OF CALIF., SANTA BARBARA
KEIO UNIVERSITY

MATH. SCIENCES RESEARCH INSTITUTE

NEW MEXICO STATE UNIV.

OREGON STATE UNIV.
Daryl Cooper

Department of Mathematics

University of California

Santa Barbara, CA 93106-3080 cooper@math.ucsb.edu

Jiang-Hua Lu

Department of Mathematics

The University of Hong Kong

Pokfulam Rd., Hong Kong

jhlu@maths.hku.hk

$$
\text { Jie Qing }
$$

Department of Mathematics

University of California

Santa Cruz, CA 95064

qing@ cats.ucsc.edu

\author{
UNIV. OF CALIF., SANTA CRUZ \\ UNIV. OF MONTANA \\ UNIV. OF OREGON \\ UNIV. OF SOUTHERN CALIFORNIA \\ UNIV. OF UTAH \\ UNIV. OF WASHINGTON \\ WASHINGTON STATE UNIVERSITY
}

These supporting institutions contribute to the cost of publication of this Journal, but they are not owners or publishers and have no responsibility for its contents or policies.

See inside back cover or msp.org/pjm for submission instructions.

The subscription price for 2016 is US $\$ 440 /$ year for the electronic version, and \$600/year for print and electronic.

Subscriptions, requests for back issues and changes of subscriber address should be sent to Pacific Journal of Mathematics, P.O. Box 4163, Berkeley, CA 94704-0163, U.S.A. The Pacific Journal of Mathematics is indexed by Mathematical Reviews, Zentralblatt MATH, PASCAL CNRS Index, Referativnyi Zhurnal, Current Mathematical Publications and Web of Knowledge (Science Citation Index).

The Pacific Journal of Mathematics (ISSN 0030-8730) at the University of California, c/o Department of Mathematics, 798 Evans Hall \#3840, Berkeley, CA 94720-3840, is published twelve times a year. Periodical rate postage paid at Berkeley, CA 94704, and additional mailing offices. POSTMASTER: send address changes to Pacific Journal of Mathematics, P.O. Box 4163, Berkeley, CA 94704-0163.

PJM peer review and production are managed by EditFLOW ${ }^{\circledR}$ from Mathematical Sciences Publishers.

PUBLISHED BY

\section{I. mathematical sciences publishers}

nonprofit scientific publishing

http://msp.org/

(C) 2016 Mathematical Sciences Publishers 


\section{PACIFIC JOURNAL OF MATHEMATICS}

Volume $283 \quad$ No. $2 \quad$ August 2016

The fundamental theorem of tropical differential algebraic geometry

FUENSANTA AROCA, CRISTHIAN GARAY and ZEINAB TOGHANI

A simple solution to the word problem for virtual braid groups

271

PaOlo Bellingeri, Bruno A. Cisneros de la CruZ and Luis

PARIS

Completely contractive projections on operator algebras

DAVID P. BLECHER and MATTHEW NEAL

Invariants of some compactified Picard modular surfaces and applications

AMIR DŽAMBIĆ

Radial limits of bounded nonparametric prescribed mean curvature surfaces

MOZHGAN (NORA) ENTEKHABI and KIRK E. LANCASTER

A remark on the Noetherian property of power series rings

BYUNG GYUN KANG and PHAN THANH TOAN

Curves with prescribed intersection with boundary divisors in moduli spaces of curves

\section{XIAO-LEI LIU}

Virtual rational Betti numbers of nilpotent-by-abelian groups

BEHROOZ MiRZAII and FATEMEH Y. MOKARI

A planar Sobolev extension theorem for piecewise linear homeomorphisms 405

EMANUELA RADICI

A combinatorial approach to Voiculescu's bi-free partial transforms

PAUL SKOUFRANIS

Vector bundle valued differential forms on $\mathbb{N} Q$-manifolds

LUCA VitAGLiANO

Discriminants and the monoid of quadratic rings 